\title{
Fuzzy Soft Groups based on Fuzzy Space
}

\author{
ABDALLAH AL-HUSBAN \\ Department of Mathematics, Faculty of Science and Technology, Irbid \\ National University, P.O. Box: 2600 Irbid, JORDAN
}

Abstract: -In this paper, a new theory of fuzzy soft groups (FSG) based on fuzzy spaces (FS) from Dib's point of view is found. The concept of FSG and fuzzy soft subgroup (FSSG) based on FS was elaborated. The relationship between FSG and FSG is also investigated.

Keywords: - FSG, FSSG, soft set (SS), fuzzy soft set (FSS).

Received: April 12, 2021. Revised: December 26, 2021. Accepted: January 20, 2022. Published: February 18, 2022.

\section{Introduction}

Explored and discussed the topic of fuzzy sets (Fsets) [1]. The fuzzy group theory evolved in the following way: The concept of a fuzzy subgroup of a group was first described in [2]. The concept of FS was suggested by Dib [3]. In the ordinary case, this idea took the place of the universal set concept. Dib [3] defined fuzzy functions (FF), fuzzy binary operations (FBO), and fuzzy subspaces (FSB) in FS. The concepts of fuzzy group, fuzzy subgroup, and fuzzy group theory were constructed in the stated study after defining FS and FBO. Begins by introducing an SS [4]. It is a parameterized family of universal set subsets and also introduces and investigates FSS [5]. It is a more general idea that combines the F-set and the SS. The goal of this research was to combine two mathematical domains on the F-set, fuzzy algebra and SS theory, to create a new algebraic system called FSG based on FS. The theory of FSG is developed and FSSG is introduced. Based on the concept of FS, the theory of SS established by [4] has been applied to fuzzy subgroups to construct the notions of FSG and FSSG.

\section{Preliminaries}

Definition 2.1 ([3]) A FS $(Q, N=[0,1])$ is the set of all ordered pairs $(q, N), q \in Q$

$(Q, N)=\{(q, N): q \in Q\}$ where $(q, N)=\{(q, n): n \in N\}$.

The ordered pair $(q, N)$ is called a fuzzy element (FE) in the FS.
Definition 2.2 ([3]) A FSB $U$ of the FS $(Q, N)$ is the collection of all ordered pairs $\left(q, \alpha_{q}\right)$ where $q \in U_{0}, \exists U_{0} \in Q$ and $\alpha_{q} \subseteq N$.

Definition 2.3 ([3]) Let $(Q, N)(Y, N)$ and $(Z, N)$ be FS. The (FF) $\underline{D}$ from $(Q, N) \times(Y, N)=(Q \times Y, N \times N)$ into $(Z, N)$ is typified with the ordered pair $\left(D, J_{q y}\right)$ where $D: Q \times Y \rightarrow Z$ a function and $J_{q y}$ is a family function $J_{x y}: N \times N \rightarrow N$ fulfilling the requirements:

(i) $J_{q y}$ is non-decreasing on $N \times N$,

(ii) $J_{q y}(0,0)=0$ and $J_{x y}(1,1)=1$.

Definition 2.4 ([3]) A FBO $\underline{D}=\left(D, J_{q y}\right)$ on the FS $(Q, N=[0,1]) \quad$ is a $\quad$ FF from $(Q, N) \times(Q, N) \rightarrow(Q, N)$ where $D: Q \times Q \rightarrow Q$ and $J_{q y}: N \times N \rightarrow N$ are functions with satisfying

(ii) $J_{q y}$ are onto.

\section{FSG over FS}


In this paper, we define (FSG) and (FSSG) and obtain some related results. Also, we will introduce FSSG induced by fuzzy soft subset.

Definition 3.1. An FSG typified by $((Q, N), \underline{D})$ is an FS iff for every, $\left(\left(q, \alpha_{F(e)}(q)\right), N\right),\left(\left(y, \alpha_{F(e)}(y)\right), N\right)$ and $\left(\left(z, \alpha_{F(e)}(z)\right), N\right) \quad$ the following requirements are met:

(i) Associative:

$$
\begin{aligned}
& \left.\left(\left(q, \alpha_{F(e)}(q)\right), N\right) \underline{D}\left(\left(y, \alpha{ }_{F(e)}(y)\right), N\right)\right) \underline{D}\left(\left(z, \alpha_{F(e)}(z)\right), N\right) \\
& =\left(\left(q, \alpha_{F(e)}(q)\right), N\right) \underline{D}\left(\left(y, \alpha_{F(e)}(y)\right), N\right) \underline{F}\left(\left(z, \alpha_{F(e)}(z)\right), N\right)
\end{aligned}
$$

It has an identity $\left(\left(e, \alpha_{F(e)}(e)\right), N\right)$, for which

$$
\begin{aligned}
& \left(\left(q, \alpha_{F(e)}(q)\right), N\right) \underline{D}\left(\left(e, \alpha_{F(e)}(e)\right), N\right) \\
& =\left(\left(\mathrm{e}, \alpha_{F(e)}(x)\right), N\right) \underline{D}\left(\left(q, \alpha_{F(e)}(q)\right), N\right) \\
& =\left(\left(q, \alpha_{F(e)}(q)\right), N\right),
\end{aligned}
$$

(ii) Every fuzzy soft element (FSE) $\left(\left(q, \alpha_{F(e)}(q)\right), N\right)$ has an inverse $\left(\left(q, \alpha_{F(e)}(q)\right), N\right)^{-1}$ such that

$$
\begin{aligned}
& \left(\left(q, \alpha_{F(e)}(q)\right), N\right) \underline{D}\left(\left(q, \alpha_{F(e)}(q)\right), N\right)^{-1} \\
& =\left(\left(q, \alpha_{F(e)}(q)\right), N\right)^{-1} \underline{D}\left(\left(q, \alpha_{F(e)}(q)\right), N\right) \\
& =\left(\left(q, \alpha_{F(e)}(q)\right), N\right) .
\end{aligned}
$$

Denote $(q, N)^{-1}=(q, N)$, then

$$
\begin{aligned}
& \left(\left(q D y, \alpha_{F(e)}(q D y)\right), N\right) \\
& =\left(\left(y D q, \alpha_{F(e)}(q)\right), N\right) \\
& =\left(\left(e, \alpha_{F(e)}(e)\right), N\right)
\end{aligned}
$$

It follows from (i), (ii), and (iii) which $(Q, \underline{D})$ is an FSG.

Theorem 3.1 Associated to each FSG $((Q, N), \underline{D})$ there is a fuzzy group $((Q, N), \underline{D})$ and they are isomorphic to each other by the correspondence $\left(\left(q, \alpha_{F(e)}(q)\right), N\right) \leftrightarrow(q, N)$.

Definition 3.2 A FSG $((Q, N), \underline{D})$ is said to be a commutative FSG if

$\left(\left(q, \alpha_{F(e)}(q)\right), N\right) \underline{D}\left(\left(y, \alpha_{F(e)}(y)\right), N\right)$

$=\left(\left(y, \alpha_{F(e)}(y)\right), N\right) \underline{D}\left(\left(q, \alpha_{F(e)}(q)\right), N\right)$

for all FSE $\left(\left(q, \alpha_{F(e)}(q)\right), N\right)$ and $\left(\left(y, \alpha_{F(e)}(y)\right), N\right)$ of the FS $(Q, N)$.

Example 3.2 Consider the set $C=\square_{4}=\{0,1,2,3\}$, $E=\{0,1,2\}$. Define FBO $\underline{D}=\left(D, J_{q y}\right)$ over the FS $(\square, N=[0,1])$ set as follow:

$D(q, \mathrm{y})=q+_{4} y, \quad$ where $t_{4}$ refers to addition modulo 4 ,

$$
J_{x y}(n, m)=n \wedge m \text { and }
$$

$\tilde{F}: \square_{4} \rightarrow N^{A}, \tilde{\mathrm{F}}(\mathrm{e}): C \rightarrow N=[0,1]$.

$$
\begin{aligned}
& (\tilde{F}, C)=\left\{\alpha_{F(e)}(0)=\left\{\frac{0}{1}, \frac{1}{1}, \frac{2}{1}, \frac{3}{1}\right\},\right. \\
& \alpha_{F(e)}(1)=\left\{\frac{0}{0.5}, \frac{1}{0}, \frac{2}{0}, \frac{3}{0}\right\}, \\
& \alpha_{F(e)}(2)=\left\{\frac{0}{0.8}, \frac{1}{0.8}, \frac{2}{0.8}, \frac{3}{0.8}\right\}, \\
& \left.\alpha_{F(e)}(3)=\left\{\frac{0}{0.2}, \frac{1}{0}, \frac{2}{0.2}, \frac{3}{0}\right\}\right\} .
\end{aligned}
$$

The element of FSS $\alpha_{F(e)}(0)=\left\{\frac{0}{1}, \frac{1}{1}, \frac{2}{1}, \frac{3}{1}\right\}$. Thus $\left.\left(\square{ }_{4}, N\right), \underline{D}\right)$ is an FSG.

Theorem 3.2 The statements are correct for any FSG:

(1) For fuzzy soft identity, the element is unique.

(2) The inverse of each FSE

$$
\left(\left(q, \alpha_{F(e)}(q)\right), N\right) \in((Q, N), \underline{D}) \quad \text { is }
$$
unique.

$$
\begin{aligned}
& \left(\left(\left(q^{-1}\right)^{-1}, \alpha_{q D(e)}\left(q^{-1}\right)^{-1}\right), N\right) \\
& =\left(\left(q, \alpha_{q D(e)}(q)\right), N\right) .
\end{aligned}
$$


Definition 3.3. Let $((Q, N=[0,1]), \underline{D})$ be an FSG and let $U=\left\{\left(q, \alpha_{q}\right): q \in U_{0}\right\}$ be an FSB of $(Q, N) .(U, \underline{D})$ is called an FSSG of the FSG $((Q, N), \underline{D})$ if:

(i) $\quad \underline{D}$ is closed on the FSB $U$, i.e.,

$$
\begin{aligned}
& \left(q, \alpha_{q}\right) \underline{D}\left(y, \alpha_{y}\right) \\
& =\left(q D y, \alpha_{q D y}\right) \\
& =\left(q D y,\left(\alpha_{q} J_{q y} \alpha_{y}\right)\right)
\end{aligned}
$$

(ii) $\quad(U, \underline{D})$ meets the requirements of an FSG

Theorem 3.3 $(U, \underline{D})$ is an FSSG of the FSG $((Q, N=[0,1]), \underline{D})$ if and only if:

(1) $\left(U_{0}, D\right)$ is a subgroup of $(Q, D)$,

(2) $J_{q y}\left(\alpha_{q}, \alpha_{y}\right)=\alpha_{x D y}=\left(\alpha_{q} J_{q y} \alpha_{y}\right)$.

Proof. If (1) and (2) are satisfied, then

(a) $\underline{D}$ is closed on the FSB $U$. Let $\left(q, \alpha_{q}\right),\left(y, \alpha_{y}\right) \in U$. Then

$$
\begin{aligned}
& \left(q, \alpha_{q}\right) \underline{D}\left(y, \alpha_{y}\right) \\
& =\left(q D y, J_{q y}\left(\alpha_{q}, \alpha_{y}\right)\right) \\
& =\left(q D y, \alpha_{q D y}\right) \in U .
\end{aligned}
$$

(b) $(U, \underline{D})$ satisfies the FSG. Let $\left(q, \alpha_{q}\right)$, $\left(y, \alpha_{y}\right),\left(z, \alpha_{z}\right) \in U$.Then (b1)

$$
\begin{aligned}
& \left(( q , \alpha _ { q } ) \underline { D } ( y , \alpha _ { y } ) \left(\underline{D}\left(z, \alpha_{z}\right)\right.\right. \\
= & \left(q D y, \alpha_{q D y}\right) \underline{D}\left(z, \alpha_{z}\right) \\
= & \left((q D y) D z, \alpha_{(q D y) D z}\right) \\
= & \left(q D(y D z), \alpha_{q D(y D z)}\right) \\
= & \left(q, \alpha_{q}\right) \underline{D}\left(y D z, \alpha_{y D z}\right) \\
= & \left(q, \alpha_{q}\right) \underline{D}\left(\left(y, \alpha_{y}\right) \underline{D}\left(z, \alpha_{z}\right) .\right.
\end{aligned}
$$

$$
\begin{aligned}
\left(q, \alpha_{q}\right) \underline{D}\left(e, \alpha_{e}\right) & =\left(q D e, \alpha_{q D e}\right) \\
& =\left(q, \alpha_{q}\right) \\
& =\left(e D q, \alpha_{e D q}\right) \\
& =\left(e, \alpha_{e}\right) \underline{D}\left(q, \alpha_{e}\right) .
\end{aligned}
$$

(b3) Each $\left(q, \alpha_{q}\right)$ has an inverse $\left(q^{-1}, \alpha_{q^{-1}}\right)$, since

$$
\begin{aligned}
\left(q, \alpha_{q}\right) \underline{D}\left(q^{-1}, \alpha_{q^{-1}}\right) & =\left(q D q^{-1}, \alpha_{q D q^{-1}}\right) \\
& =\left(q^{-1} D q, \alpha_{q^{-1} D q}\right) \\
& =\left(e, \alpha_{e}\right) \\
& =\left(q^{-1}, \alpha_{q^{-1}}\right) \underline{D}\left(q, \alpha_{q}\right) .
\end{aligned}
$$

We can deduce from (a) and (b) that $(U, \underline{D})$ is an FSSG of FSG $((Q, N), \underline{D})$. Conversely if $(U, \underline{D})$ is an FSSG of FSG $((Q, N), \underline{D})$ Then (i) is restricted by associativity. The following is also valid:

$$
\begin{aligned}
\left(\alpha_{q} J_{q y} \alpha_{y}\right) & =J_{q y}\left(\alpha_{q} \times \alpha_{y}\right) \\
& =\alpha_{q D y} .
\end{aligned}
$$

Theorem 3.4 $(U ; \underline{D})$ is an FSSG of the FSG $((Q, N=[0,1]), \underline{D})$ iff:

(i) $\quad q D y^{-1} \in U_{0}$, for every $q, y \in U_{0}$;

(ii) $J_{q y}\left(\alpha_{q}, \alpha_{y}\right)=\alpha_{q D y}=\left(\alpha_{q} J_{q y} \alpha_{y}\right)$. 
Theorem 3.5 let $\left(H_{0}(C), \underline{D}\right),(\underline{H}(C), \underline{D})$ and $(\bar{H}(C), \underline{D})$ are FSSG of the FSG $((Q, N), \underline{D})$ if;

(i) $\left(C_{0}, D\right)$ is a subgroup of $(Q, D)$ where $C_{\mathrm{o}}=\{q \in Q: C(q) \neq 0\}$;

$$
J_{x y}(\underline{C}(q), \underline{C}(\mathrm{y}))=C(q d y), \forall q, y \in C_{0} .
$$

Proof. We prove the result for $\left(H_{0}(C), \underline{D}\right)$ and the rest would be done in the same way.

Let $\left(q, \alpha_{q}\right)=H_{0}\left(\alpha_{q}\right)$ and let be $\left(H_{0}\left(\alpha_{q}\right), \underline{D}\right)$ an $F S S G$ of the FSG $((Q, N), \underline{D})$. Then (i) holds by Again by Theorem 3.2 we have:

$$
\begin{aligned}
J_{q y}\left(\alpha_{q}, \alpha_{y}\right) & =J_{q y}\left(\left(0, \alpha_{q}\right),\left(0, \alpha_{y}\right)\right) \\
& =\alpha_{q D y} \\
& =\left(0, \alpha_{q F y}\right)
\end{aligned}
$$

That is, $J_{q y}\left(\left(0, \alpha_{q}\right),\left(0, \alpha_{y}\right)\right)=J_{q y}\left(\left(0, \alpha_{q}\right),\left(0, \alpha_{y}\right)\right)$

$$
=u_{q D y} \text {. }
$$

Conversely, assume that conditions (i) and (ii) hold, then:

$$
\begin{aligned}
J_{q y}\left(\left(0, \alpha_{q}\right),\left(0, \alpha_{y}\right)\right) & =J_{q y}\left(\left(0, \alpha_{q}\right),\left(0, \alpha_{y}\right)\right) \\
& =J_{q y}\left((0,0), J_{q y}\left(0, \alpha_{q}\right),\left(0, \alpha_{y}\right)\right) \\
& =\alpha_{q D y} .
\end{aligned}
$$

Similarly, we can prove that $J_{q y}\left(\left(0, \alpha_{q}\right),\left(0, \alpha_{y}\right)\right)=\alpha_{q D y}$ which proves by Theorem 3.2 that $\left(H_{0}(C), \underline{D}\right)$ is fuzzy subgroups of $((Q, N), \underline{D})$.

\section{Conclusion}

In this paper, we have applied the fuzzy group study started by Dib (1994) to the context of FSG.

\section{References:}

[1] Zadeh, L.A, Fuzzy Sets, Inform. Control Vol.8, 1965 , pp. 338-353.
[2] Rosenfeld, A, Fuzzy Groups. Journal of Mathematical Analysis and Applications Vol. 35, 1971, pp. 512-517.

[3] Dib, K. A. On Fuzzy Spaces and Fuzzy Group Theory. Information Sciences, Vol. 80, 1994, pp. 253-282.

[4] Molodtsov, D, Soft Set Theory First Result. An International Journal of Computers and Mathematics with Applications, Vol. 37, 1999, pp. 19-31.

[5] Maji, P. K., Biswas, R, \& Roy, A.R. Fuzzy Soft Set Theory. The Journal of Fuzzy Mathematics Vol. 9(3), 2001, pp. 589-602.

[6] Dib, K.A. \& Hassan, A.A.M. The fuzzy Normal Subgroup. Fuzzy Sets and Systems. Vol. 98, 1998, pp.393-402.

[7] Akta. H. Gagman N. Soft Sets and Soft group, Information Science Vol. 177, 2007, pp.27262735.

[8] Selvachandran, G. Fuzzy Soft Rings Based on Fuzzy Spaces. International Journal of Mathematical Analysis, 8(56), 2014, pp. 27812787.

[9] Jaradat, A., \& Al-Husban, A The Multi-Fuzzy Group Spaces on Multi-Fuzzy Space. J. Math. Comput. Sci, Vol. 11(6), 2021, pp.7535-7552.

[10] Rosenfeld A, Fuzzy Groups, Math .Anal .Appl. Vol. 35, 1971, pp. 512-517.

[11] Anthony, J. M. \& Sherwood, H. A Characterization of Fuzzy Subgroups. Fuzzy Sets and Systems Vol. 7, 1982, pp. 297-305.

[12] Pazar. B.V \& Aygunoglu. A \& Ygun. H. On Fuzzy Soft Rings. Journal of Hyper structures Vol. 1(2), 2012, pp.1-15.

[13] Das, P. S. Fuzzy Groups and Level Subgroups. Journal of Mathematical Analysis and Applications. Vol. 84, 1981, pp.264-269.

[14] Dib, K. A. \& Youssef, N. L. Fuzzy Cartesian Product, Fuzzy Relations and Fuzzy Functions. Fuzzy Sets and Systems. Vol. 41, 1991, pp. 299-315.

[15] Abdallah Al-Husban \& Abdul Razak Salleh. Complex Fuzzy Group based on Complex fuzzy space. Global Journal of Pure and Applied Mathematics. Vol. 12(2), 2016, pp. 1433-1450.

[16] Abdallah Al-Husban, Abdul Razak Salleh, \& Nasruddin Hassan, Complex Fuzzy Normal Subgroup." THE ukm fst postgraduate colloquium: Proceedings of the Universiti Kebangsaan Malaysia, Faculty of Science and Technology 2015 Postgraduate Colloquium. Vol. 1678. , 2015, pp. 060008-060015. 
[17] Abdallah Al-Husban \& Abdul Razak Salleh, Complex Fuzzy Ring. Proceedings of 2nd International Conference on Computing, Mathematics and Statistics. PP. 241-245. Publisher: IEEE 2015.

[18] Abdallah Al-Husban \& Abdul Razak Salleh 2015. Complex Fuzzy Hyperring Based on Complex Fuzzy Spaces. Proceedings of 2nd Innovation and Analytics Conference \& Exhibition (IACE). Vol. 1691, 2015, pp. 040009-040017.

[19] Abdallah Al-Husban \& Abdul Razak Salleh. Complex Fuzzy Hypergroup based on Complex Fuzzy Space. International Journal of Pure and Applied Mathematics. Vol. 107(4), 2016, pp. $949-958$.

[20] Al-Husban, A., Amourah, A., \& Jaber, J. J. Bipolar complex fuzzy sets and their properties. Italian Journal of Pure and Applied Mathematics, N (43) 2020, pp. 754-761.

[21] Alsarahead, MIKHLED O., and A. G. Ahmad. "Complex Fuzzy Soft Group." Journal of Quality Management and Analysis vol.13.2, 2017, pp. 17-28.

[22] Riaz, M., Saeed, M., Saqlain, M., \& Jafar, N. Impact of water hardness in instinctive laundry system based on fuzzy logic controller. Punjab University Journal of Mathematics, 2020, 51(4).

[23] Luqman, A., Akram, M., Al-Kenani, A. N., \& Alcantud, J. C. R. (2019). A study on hypergraph representations of complex fuzzy information. Symmetry, Vol. 11(11), 2019, pp. 1381.

[24] Al Tahan, M., \& Davvaz, B. (2018). Generalized Complex Fuzzy and Anti-Fuzzy H_v-Subgroups. International Journal of Analysis and Applications, 16(5), 2018, pp.628-642.
Sources of Funding for Research Presented in a Scientific Article or Scientific Article Itself

No fund

\section{Creative Commons Attribution License 4.0}

(Attribution 4.0 International, CC BY 4.0)

This article is published under the terms of the Creative Commons Attribution License 4.0 https://creativecommons.org/licenses/by/4.0/deed.en UShttps://creativecommons.org/licenses/by/4.0/dee d.en_US 